\section{ANALYSIS OF TORQUE VERTICAL ON STRING MODEL}

Ari Kusumastuti*, Sri Sasi Yuni Nurhayati

Department of Mathematics, State Islamic University of Maulana Malik Ibrahim, Malang, Indonesia
Article history

Received

4 July 2015

Received in revised form

2 November 2015

Accepted

3 March 2016

*Corresponding author arikusumastuti@gmail.com

\section{Graphical abstract}

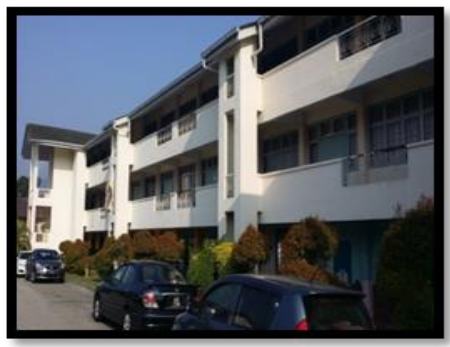

\begin{abstract}
Different to other motion of torque, motion the torque modeled by McKenna (1999) is the motion of torque into a vertical direction, a consequence of the imposition of downward. Mckenna lowering the model of two pieces of rope in fastening on a beam then in hanging, then McKenna replaces the parameters with forensic data from Tacoma bridge, to analyze a wave of what happened at the bridge collapse that resulted in the bridge. In this research will be shown that the solution of the equation of vertical torque from the results of having a conspicuous disparity linearization with a numerical solution
\end{abstract}

Keywords: Oscillation, torque, numerical analysis

(C) 2016 Penerbit UTM Press. All rights reserved

\subsection{INTRODUCTION}

In year 1999, lazer McKenna and managed to formulate a model of the mathematics of one example of the phenomenon of creeping vibration on stringed [1]. Lazer McKenna and formulate a model of the motion of torque and vertical of two pieces of rope that bind a beam. Then of the model and obtained Lazer McKenna replace the parameters of the report with forensic of the Tacoma. Study on the motion of the torque is very important because many tool apply the motion of torque, like a spring, pendulum and others .In addition to a bridge motion torque also occurred in high-rise buildings due to wind and also the quake as well as the current return of an electric generator. Because it is very important to do an analysis of behavior from a deflection formed from the motion of this torque, mckenna the model was formulated by tying a beam on two hanging rope. As in Figure 1

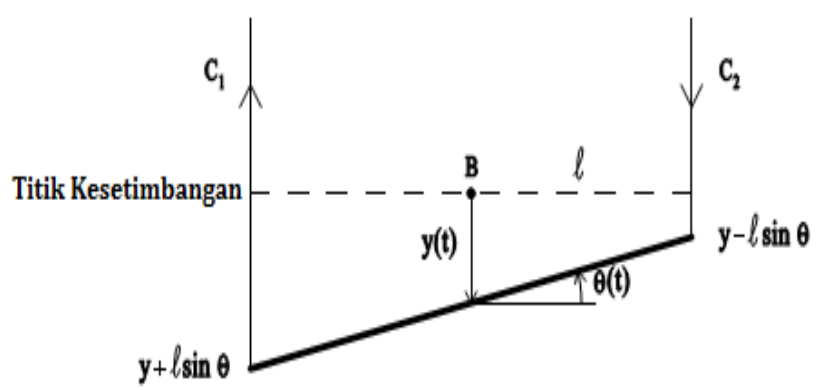

Figure 1 Vertical torque motion of string

So obtained not paired system as follows:

$$
\begin{aligned}
& y=-\frac{6 K}{m} \cos \theta \cdot \sin \theta-\delta_{1} \theta+f(t) \\
& y=-\frac{2 K}{m} y-\delta_{2} y+g(t)
\end{aligned}
$$

$\theta(t)$ is the angle of horizontal on stringed at certain times in call with the angle of deflection. While $y(t)$ is the dynamics of the vertical movement of stringed suspension. 


\subsection{LITERATURE REVIEW}

\subsection{McKenna Model}

A differential equation uncoupled obtained from [1] to torque motion and vertical beam with the assumption that vertical wire never lost voltage that has been granted. McKenna shows that the period of the same small, the size of the the periodic movement of amplitude, the final result depends on the initial conditions [2].

The force used by stringed is proportional to lengthening on stringed, in Figure 1, indicated that extra stringed part right is $(y-l \sin \theta)$ Hence the force used is:

$$
\begin{gathered}
-K(y \pm l \sin \theta)^{+}= \begin{cases}-K(y \pm l \sin \theta), & y \pm l \sin \theta \geq 0 \\
0 & y \pm l \sin \theta<0\end{cases} \\
\left\{\begin{array}{l}
\tilde{\theta}=\frac{3 K}{m l} \cos \theta\left[(y-l \sin \theta)^{+}-(y+l \sin \theta)^{+}\right]-\hat{\sigma}_{2} \theta+f(t) \\
\ddot{y}=-\frac{K}{m}\left[(y-l \sin \theta)^{+}+(y+l \sin \theta)^{+}\right]-\hat{\sigma}_{2} y+g
\end{array}\right.
\end{gathered}
$$

A differential equation uncoupled obtained a writer in (McKenna, 1999), to torsional motion and vertical beams with the assumption that wire vertical never lost a voltage have been given. So the equation (2) becomes:

$$
\begin{aligned}
& \dot{\theta}=-\frac{6 K}{m} \cos \theta \sin \theta-\delta_{1} \theta+f(t) \\
& y=-\frac{2 K}{m} y-\delta_{2} y+g
\end{aligned}
$$

\subsection{Order Reduction of ODE}

Given second order of ordinary differential equation $\ddot{x}=-k x-b \dot{x}$

e.g. $\mathrm{x}_{1}=x$ and $x_{2}=\dot{x}$, So the equation (4) of differential equations can be written as a first order of linear system

$$
\begin{aligned}
& x_{1}=x_{2} \\
& x_{2}=-k x_{1}-b x_{2}
\end{aligned}
$$

Or in the form of a matrix as follows:

$$
\boldsymbol{x}=\left(\begin{array}{cc}
0 & 1 \\
-k & -b
\end{array}\right) \boldsymbol{x}
$$

\subsection{Predictor Corrector Methods}

A method of predictor-corrector is an assemblage of two parallels of to $Y_{n+1}$ An equation first, called predictor, used to predict (obtain of approximating to) $Y_{n+1}$. Second equation, called a corrector, then used to obtain the value the results of correction $y_{n+1}$. In general a corrector depend on the value of that predicted.

Predictor : Approximating the $Y_{\mathrm{r}+1}$ from $Y_{T}, Y_{T-1}, Y_{T-2}, \ldots$
Corrector: Improve the value of $\mathrm{V}_{\mathrm{r}+1}$ from predictor.

One of the scheme used in the methods of predictor a corrector are a schema of adams bashforth moulton .

$$
\text { Predictor }
$$$$
y *_{\gamma+1}=y_{\gamma}+\frac{h}{24}\left(-9 f_{y-1}+37 f_{y-2}-59 f_{\gamma-1}+55 f_{Y}\right)
$$

Corrector :

$y_{\gamma+1}=y_{\gamma}+\frac{h}{24}\left(f_{\gamma-2}-5 f_{\gamma-1}+19 f_{Y}+9 f *_{\gamma+1}\right)$

\subsection{METHODOLOGY}

Method of research is conducted in this paper uses literature study. After retrieving the data from previous studies is still a second-order ordinary differential equations that must be reduced to first become a first-order ordinary differential equations. After tlis step it can be executed by using Bashforth Adams Moulton and obtain numerical results. And then compared with the results of linearization.

\subsection{RESULTS AND DISCUSSION}

Because in the study assumed the absence of external factors that affect the vibrations, so $\mathrm{f}(\mathrm{t})=0$. So will be obtained:

$\ddot{\theta}=-\frac{6 K}{m} \cos \theta \sin \theta-\delta \theta$

After done simplification then be obtained:

$$
\theta=-\frac{3 K}{m} \sin 2 \theta-\delta \theta
$$

With order reduction of ODE, the equation (8) will be obtained:

e.g. $\theta_{1}=\theta$ and $\theta_{2}=\theta$,

After derivate $\theta_{1}$ and $\theta_{2}$ to $t$, so will be obtained $\tilde{\theta}_{1}=\tilde{\theta}$ and $\tilde{\theta}_{2}=\ddot{\theta}$. Consequently equation (7) turned into system as follows

$\left\{\begin{array}{l}\theta_{1}=\theta_{2} \\ \theta_{2}=-\frac{3 K}{m} \sin 2 \theta_{1}-\delta \theta_{2}\end{array}\right.$

Because $\tilde{\theta}_{2}$ is nonlinear because of the form of a sinus, hence the form of a sinus done expansion by using the taylor around a point $(0,0)$ until cutting first order, to be obtained

$$
\begin{aligned}
\sin 2 \theta_{1}= & \sin (2 \cdot 0)+\frac{d}{d \theta} \sin (2 \cdot 0) \cdot\left(\theta_{1}-0\right) \\
& =2 \theta_{1}
\end{aligned}
$$

So that will be gained by a new system that is: 
$\boldsymbol{\theta}=\left(\begin{array}{cc}0 & 1 \\ -\frac{6 K}{m} & -\delta\end{array}\right) \cdot \boldsymbol{\theta}$

In the same way to obtain solution of the equation vertical motion done being a system of linear reduction .By ignoring gravitation: style to be obtained:

$y=-\frac{2 K}{m} y-\delta_{2} y$

Of the equation ( 11 ) then reduced to a system of linear follows:

e.g. $y_{1}=y$ and $y_{2}=\dot{y}$. Then:

$\left\{\begin{array}{c}y_{1}=y_{2} \\ y_{2}^{*}=-\frac{2 \mathrm{~K}}{\mathrm{~m}} \mathrm{y}_{1}-\delta \mathrm{y}_{2}\end{array}\right.$

By determining eigen values and eigen vectors eat solution be obtained from the system:

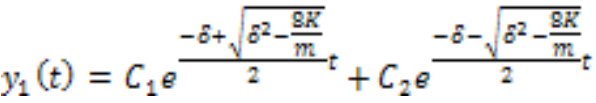

$y_{2}(t)=\frac{-\delta+\sqrt{\delta^{2}-\frac{8 K}{m}}}{2} C_{1} e^{\frac{-\delta+\sqrt{\delta^{2}-\frac{g K}{m}}}{2}}+\frac{-\delta-\sqrt{\delta^{2}-\frac{8 K}{m}}}{2} \cdot C_{2}$

Figure 2 shows the plot of torque equation linierized with $\theta_{1}(0)=1.2$ and $\theta_{2}(0)=0$. Figure 3 shows the plot of adams bashforth moulton of torque equation with $\theta_{1}(0)=1.2$ and $\theta_{2}(0)=0$. On the other hand, Figure 4 visualizes the plot of vertical motion equation with $Y_{1}(0)=1$ and $Y_{2}(0)=1$ and finally Figure 5 demonstrates the plot of adams bashforth moulton of vertical motion equation with $Y_{1}(0)=1$ and $Y_{2}(0)=1$

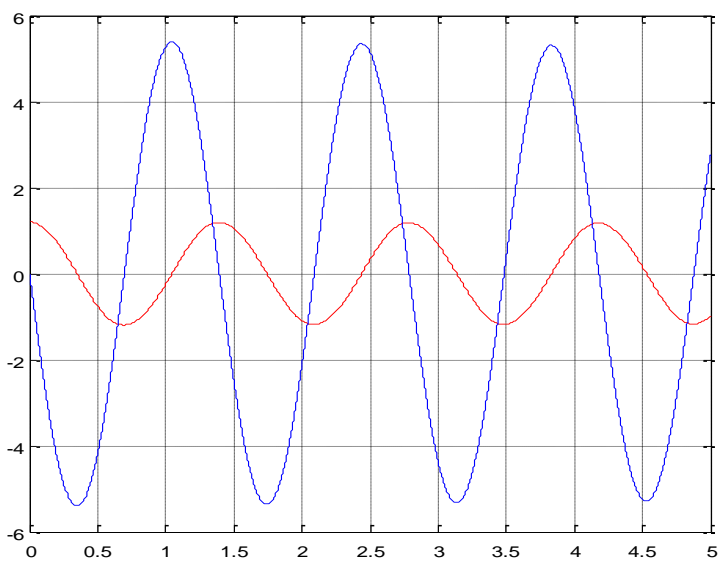

Figure 2 Plot of torque equation linierized with $\theta_{1}(0)=1.2$ and $\theta_{2}(0)=0$

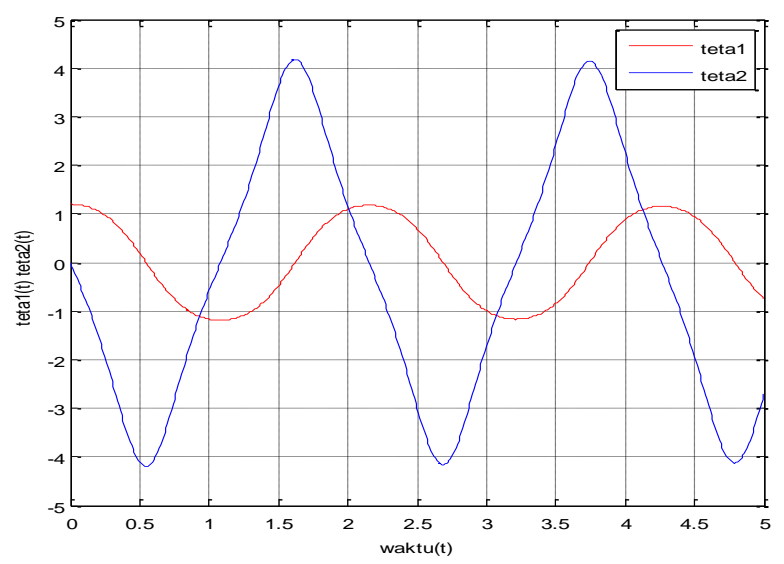

Figure 3 Plot of adams bashforth moulton of torque equation with $\theta_{1}(0)=1.2$ and $\theta_{2}(0)=0$

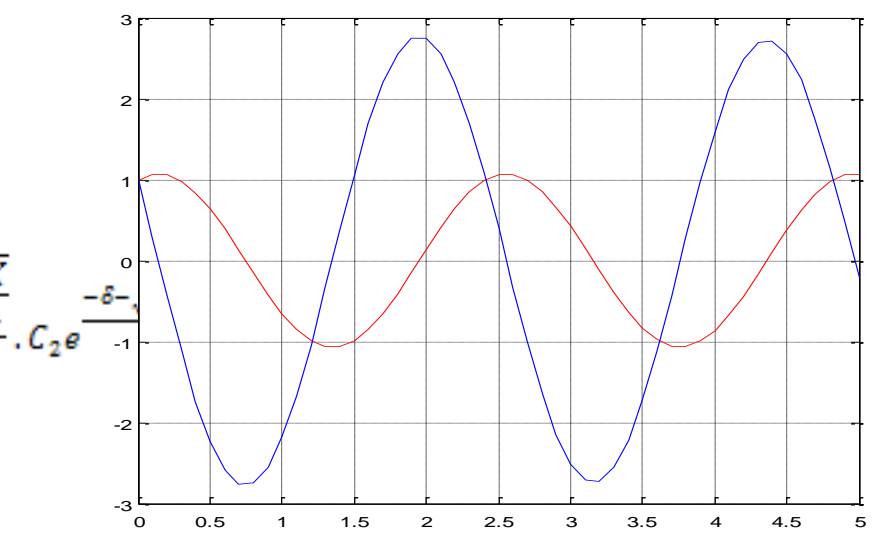

Figure 4 Plot of vertical motion equation with $y_{1}(0)=1$ and $Y_{2}(0)=1$

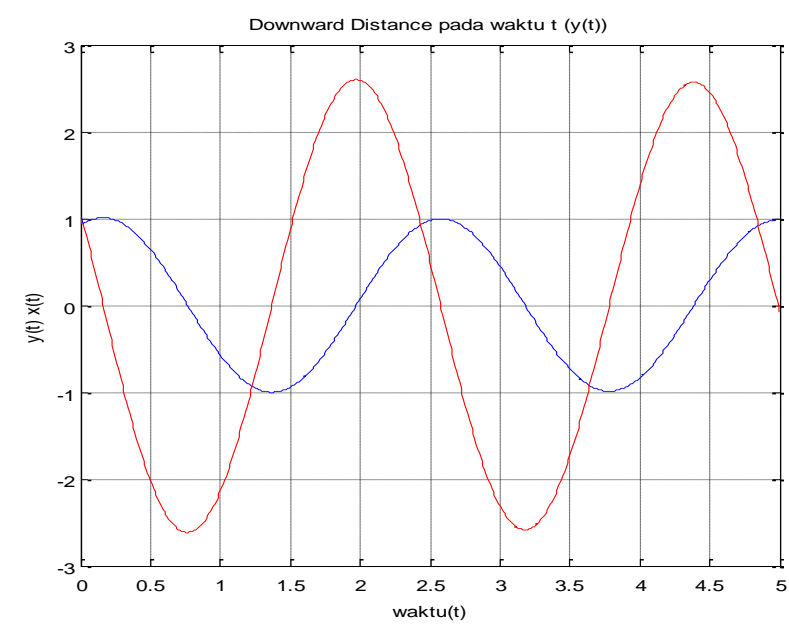

Figure 5 Plot of adams bashforth moulton of vertical motion equation with $Y_{1}(0)=1$ and $V_{2}(0)=1$ 
The results of a solution in the equation linearization torque that has been obtained, an initial value given not give a big influence on the size of the amplitude on waves .Because whatever the value of the beginning of a start given, long wave will remain muted to the point of $\left(0_{2}, 0\right)$ and finally stop, if initial value given large enough give the effect of damping given the small, but underwent will still stop. But of numerical analysis using a method of adams bashforth linearization without doing a solution having a big difference, the result of rapid linearization precatorius waves more quickly compared with the result of a method of adams bashforth. From the results of a method of adams bashforth also an initial value different had an impact that large, so if the value of a start given different, solutions of an equation torque not necessarily converging to a point $(0,0)$. While on similarities vertical motion the results of a solution and numerical exact almost the same because linear do they have in common.

\subsection{CONCLUSION}

From discussion and plot the picture above it was concluded that the results of the solution by using linearization and using Adams Bashforth Moulton Methods has relatively similar results. So that this solution can be considered both as a solution of the above model.

\section{Acknowledgement}

In this research is still limited by the assumption that vertical wire never lost voltage that has been granted. So hopefully in future studies using the assumption that the cable ever lose voltage that has been granted

\section{References}

[1] Kwofie, R. O. 2012. A Mathematical Model Of A Suspension Bridge-Case Study: Adomi Bridge, Atimpoku, Ghana. Global Advanced Research Journal of Engineering. 047-062.

[2] Parlos, A. G. 2004. Department of Mechanical Engineering. Taken at August 17, 2014, dari Texas A\&M University College of Engineering: http://parlos.tamu.edu/MEEN65. 